\title{
BENEFICIOS DE LA IMPLEMENTACIÓN DE UN SISTEMA DE TELEMETRÍA PARA LA GESTIÓN DE LAS OPERACIONES MINERAS
}

\author{
Walter Schmidt Felsch Junior \\ walter.felsch@csn.com.br \\ Companhia Siderúrgica Nacional, Minas Gerais, Brasil \\ Carlos Enrique Arroyo Ortiz \\ carroyo@ufop.edu.br \\ Universidade Federal de Ouro Preto, Minas Gerais, Brasil \\ Valdeis de Souza Oliveira / Pablo Rodrigo Vieira da Cunha / Éder Flávio de Araújo Costa \\ valdeis.oliveira@csn.com.br / pablo.cunha@csn.com.br / eder.costa@csn.com.br \\ Companhia Siderúrgica Nacional, Minas Gerais, Brasil
}

\section{Resumen}

Este trabajo presenta los beneficios del uso de la telemetría en la gestión de la operación de una mina a cielo abierto de alta producción localizada en el estado de Minas Gerais, Brasil. Se utilizó el sistema de telemetría embarcada en los camiones Caterpillar denominado Sistema de Monitoreo de Señales Vitales (SMSV), que monitorea los camiones a través de señales de dispositivos de entrada (sensores y conmutadores) proporcionando alerta de acción a través de mensajes para el operador, utilizando un visor en la cabina. Los procesos que serán objeto de control son: suministro de combustible, desbalance de cargas, condiciones de vías de acceso y temperatura del sistema de refrigeración de los frenos. Se desarrolló una interfaz con el sistema de gestión de flotas Intellimine (Modular Mining System) para la gestión de las operaciones mineras. Se implementó un sistema que identifica el tipo del problema generado y los medios más eficientes para su resolución y localización de las anomalías en tiempo real.

Palabras clave: telemetría, minería, gestión, equipo, reducción de costos

Abstract

\section{Benefits of implementing a telemetry system for mining operations management}

This research presents the benefits of using telemetry in the operations management of a high-production open-pit mine located in the state of Minas Gerais, Brazil. Caterpillar's telemetry systems called Vital Information Monitoring System (VIMS) were used to monitor trucks via input device signals (sensors and switches), providing action alert messages for the operator through a visor in the cockpit. The processes that will be subject to control are: fuel supply, load imbalance, access conditions, and temperature of the brake cooling system. An interface was developed with the IntelliMine (Modular Mining Systems) fleet management system for mining operations management. A system that identifies the type of problem was implemented, as well as the most efficient means for its resolution and localization in real time.

Keywords: telemetry, mining, management, equipment, cost reduction 


\section{Introducción}

En la actualidad los procesos productivos vienen progresivamente siendo optimizados, auxiliados por la electrónica embarcada', la informática y los sistemas de comunicación, todo lo cual permite captar datos en tiempo real y, a partir de ellos, tomar decisiones más eficaces y con mayor conocimiento técnico.

Frente a la alta variabilidad en los precios de los metales, se ha vuelto imprescindible implementar sistemas que permitan aumentar la confiabilidad y disponibilidad de los activos, reducir costos operacionales; en suma, mantener la competitividad de las empresas mineras.

Ante esta realidad, las empresas requieren incorporar como valores inherentes: competencia, creatividad, flexibilidad, agilidad, cultura de cambio y trabajo en equipo; solo así podrán mantenerse en un mercado altamente competitivo (Kardec y Nascif, 2009).

Es necesario aplicar nuevas tecnologías en la gestión de operaciones mineras, como la telemetría, a fin de que los datos recolectados puedan transformarse en información vital para mejorar los aspectos de seguridad, eficiencia y principalmente costos operacionales, ya que se reduce el margen para cometer errores e improvisar.

Lewis y Steinberg (2001) estudiaron el problema del mantenimiento de los equipos mineros en la era de la información y de su integración pues, según ellos, la gestión y monitoreo remotos permitirían reducir los costos de mantenimiento y a su vez aumentar la productividad, debido a la mayor disponibilidad física de los equipos.

El concepto de telemetría puede ser definido como la transferencia y utilización de datos originados en una red remota, posibilitando monitorear, medir y controlar las operaciones de los equipos. La comunicación puede realizarse a través de una red fija o inalámbrica. Para su implantación es necesario que existan sensores específicos correctamente instalados y personas capacitadas para analizar y ejecutar rutinas del sistema.

Existen tres aspectos tecnológicos que muestran la evolución de la telemetría:

- Tecnología de sensores, cuya característica es la captación de informaciones.

- Procesamiento y almacenamiento de la información, que mejora debido al uso de microprocesadores y memoria de gran capacidad.

- La logística para el procesamiento de la información, desde el origen al destino final.

1 Se refiere a equipos electrónicos a bordo de vehículos que utilizan circuitos integrados con funciones de sensorización y conectividad a la vez. 
De acuerdo con Knights y Daneshmend (2000), para potenciar el beneficio del uso de estas tecnologías, la industria minera debe estandarizar los datos y los protocolos de integración, lo que viabilizará el flujo de información en tiempo real, favoreciendo la toma de decisiones a nivel gerencial.

Los eventos de baja productividad y altos índices de mantenimiento de la flota de transporte son producto de varios factores. Se pueden mencionar principalmente los siguientes:

- Cargas con volumen por debajo de la capacidad de transporte.

- Condiciones críticas en el perfil de las vías, como desniveles, radios de curvatura elevados y pendientes inadecuadas.

- Límites de velocidad incompatibles en determinados tramos.

- Operaciones fuera de estándares establecidos, como cambios excesivos en el sistema de transmisión y utilización inadecuada del sistema de frenado.

Otro punto de gran importancia a considerar es la seguridad de las operaciones de mina. Las vías con perfiles acentuados o pendientes superiores al $10 \%$, poseen mayor riesgo en relación con la seguridad debido al sobrecalentamiento del sistema de refrigeración de los frenos y la consecuente pérdida de control.

La geometría de las vías de acceso debe englobar aspectos estructurales, funcionales y planos de mantenimiento, como han señalado Thompson y Visser $(1997,2000,2003,2006)$. Los costos de diseño y construcción de los accesos representan una pequeña parte comparada con los costos de mantenimiento y adecuación como los sistemas de drenaje y las inclinaciones proyectadas.

De acuerdo con Tannant y Regensburg (2001), el análisis económico en proyectos de construcción de vías de acceso es complejo pues no solo se debe tener en consideración el costo de construcción, sino también los costos de ciclo de vida completo, como:

- Costo de construcción de las vías de acceso.

- Impacto en la productividad de la flota y el costo operativo.

- Costos de operación y mantenimiento de equipos adicionales.

- Flujo de caja.

Kardec y Dorigo (2009) afirman que los responsables del mantenimiento deben garantizar la confiabilidad y disponibilidad de los activos para atender el plan de producción o prestación de servicios con seguridad, así como para la preservación del medio ambiente y el manejo de costos adecuados. 
Para Cochefski (2011), el gerenciamiento automatizado del combustible puede resultar en ahorros significativos a las empresas, así como también en el aumento de la utilización de la flota de transporte, debido a la reducción de procesos de gestión manual, ya sea por asignación de equipos o lanzamiento manual de las informaciones en hojas de control.

Por lo expuesto, el uso de la tecnología de gestión de procesos en la minería con aplicación de telemetría es evidentemente beneficioso, así como también lo es la consolidación de la información generada y su facilidad de acceso, lo que permite agilidad en la toma de decisiones.

\section{Implementación del sistema de telemetría}

El monitoreo de equipos a través de telemetría en la minería surgió debido a la carencia de procesos automáticos, a la inexistencia de controles (combustible), la calidad de vías y problemas con la operación de equipos por fuera de los estándares establecidos por los fabricantes. Estas condiciones pueden ocasionar fallas prematuras en componentes, lo que impacta directamente en el rendimiento durante su vida útil, perjudicando así su productividad, calidad y, principalmente, lo que fue programado por la empresa. Se identificaron los siguientes problemas en las operaciones y mantenimiento a considerar en la implementación:

Problemas de operación de mina:

- Frecuencia de abastecimiento con bajo volumen de combustible diésel.

- Control inexistente de las condiciones de las vías de acceso.

- Cargas transportadas en posición desbalanceada.

- Fallas en la comunicación entre los equipos.

- Falta de concentración de la información.

Problemas de mantenimiento de mina:

- Alto número de eventos de sobrecalentamiento del sistema de refrigeración de los frenos.

- Daños en el chasis y desgastes prematuros en neumáticos.

- Exceso de mantenimiento no programado.

- Fallas prematuras en cilindros y suspensiones.

- Fallas reincidentes por no existir detección de causa raíz. 
El monitoreo comienza con la recolección de los datos recibidos por el Sistema de Monitoreo de Señales Vitales (SMSV), tecnología electrónica embarcada en los camiones mineros Caterpillar que es responsable del monitoreo avanzado y el diagnóstico, y que tiene como objetivo evaluar y gerenciar la "salud" de los equipos y su rendimiento.

EI SMSV monitorea el equipo a través de señales de dispositivos de entrada (sensores y conmutadores) y proporciona alertas de acción a través de mensajes para el operador, utilizando un visor en la cabina (advisor). También tiene como función almacenar datos y registrar las condiciones del equipo para que se realicen el seguimiento y diagnósticos de problemas. La figura 1 ilustra el flujo operativo propuesto en el trabajo.
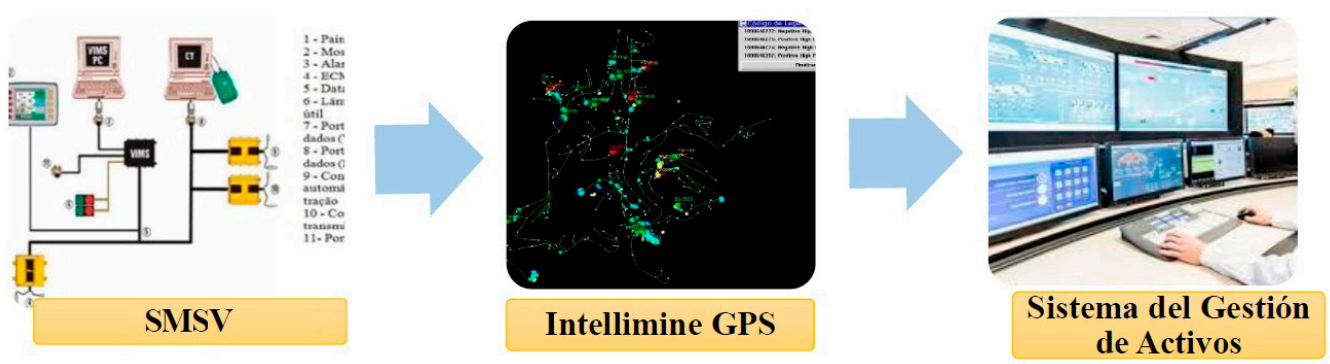

Figura 1. Corriente de información y sistemas utilizados para la gestión de procesos Elaboración propia

Gracias a la interacción con el sistema Intellimine, responsable del direccionamiento de los equipos de producción de la mina, y el SMSV, fue posible establecer una interfaz que permite detectar problemas e identificar su posición geográfica. Estas informaciones son transferidas a una central de control que monitorea los eventos y las dirige a los gestores responsables del mantenimiento y operación para el análisis y resolución de los problemas identificados.

Los principales procesos que serán monitoreados en este trabajo son:

- Procesos operativos: abastecimiento, condiciones de vías de acceso y centralización de las cargas transportadas.

- Mantenimiento: temperatura del aceite de refrigeración de los frenos. 


\subsection{Control de abastecimiento}

Son tres las opciones para el control del abastecimiento en los equipos de mina:

- Control de abastecimiento manual (vía operadores de equipos): el operador identifica la necesidad de abastecimiento y solicita liberación para desplazamiento al puesto de combustible.

- Control de abastecimiento automático (vía control de combustible): el sistema de despacho utiliza una lógica de decrecimiento de combustible a través de la inserción del consumo medio (litros/hora) y del número de horas operadas por cada equipo.

- Control de abastecimiento online (vía telemetría): El sistema de despacho realiza la lectura automática de la red CAN (Controller Area Network) del equipo; extrae en tiempo real la información de volumen de diésel y realiza la asignación del equipo al local de abastecimiento.

El objetivo fue reducir los costos operacionales a través de la reducción de la frecuencia de abasto de los equipos y la reducción de desplazamientos y colas en el puesto de combustible, permitiendo un aumento de la utilización efectiva de la flota.

El análisis consistió en migrar del control de abastecimiento automático al online, que utiliza los sistemas SMSV e Intellimine. Fue posible acompañar en tiempo real los niveles de consumo de combustible por los técnicos directamente en la sala de control. La figura 2 muestra la interfaz gráfica que acompaña el consumo y direcciona los equipos al puesto de combustible.

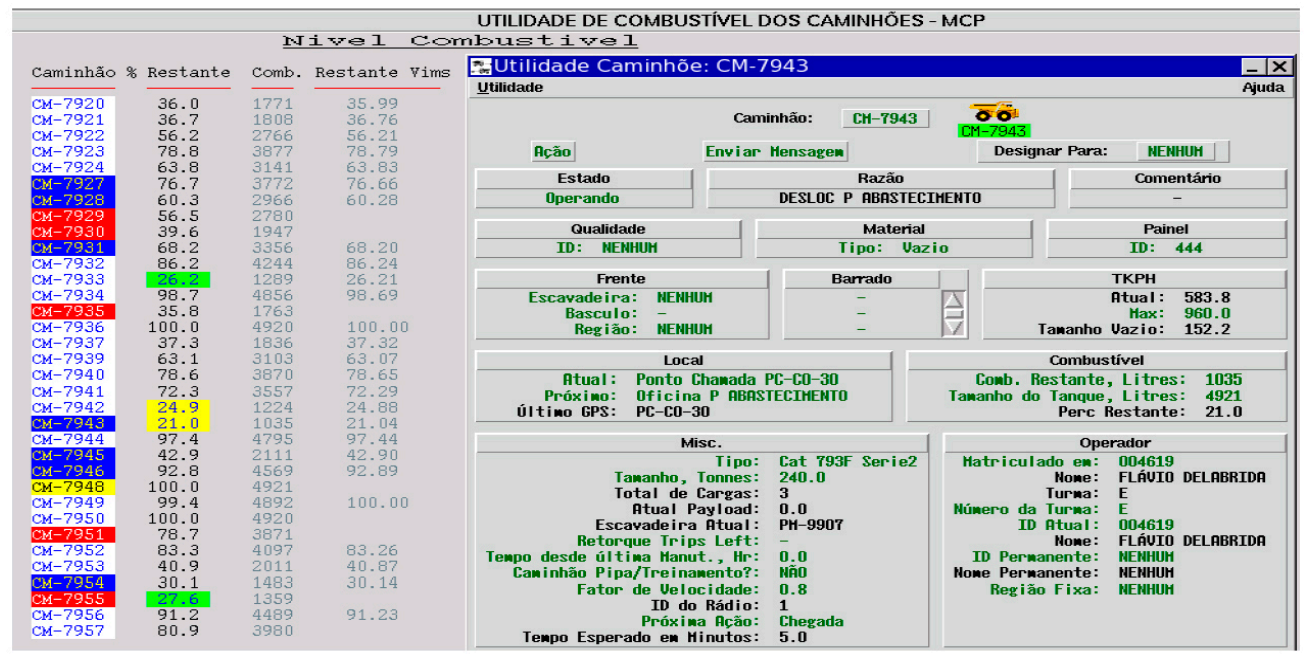

Figura 2. Interfaz de seguimiento en línea del volumen de diésel de los equipos 
Después de cuatro meses de implementado este sistema, se observaron mejoras significativas en relación con el aumento de volumen medio abastecido de la flota, además de la reducción de la variabilidad del muestreo en el abastecimiento de combustible. También fue posible crear un indicador de rendimiento denominado "volumen de abastecimiento", indicador de mucha utilidad para el control gerencial mensual.

\subsection{Monitoreo de las vías de acceso por la herramienta RAC}

Este monitoreo tiene como finalidad cuidar la "salud" de los equipos, es decir, evitar que componentes como neumáticos, suspensión, cilindros y chasis de los camiones queden fuera de servicio debido a las malas condiciones de las vías, y que cumplan con la vida útil prevista por los fabricantes.

El RAC (Road Analysis Control) es una tecnología de información a bordo que forma parte del SMSV. Fue diseñado para medir la calidad de las vías pues informa en tiempo real y de forma continua las condiciones de las vías, permitiendo identificar puntos o locales perjudiciales para los ciclos productivos y los componentes de mantenimiento. Por medio de un computador a bordo, situado en la cabina de los equipos, dos tipos de eventos del RAC avisan al operador sobre trechos de las vías que requieren cuidado, tanto del operador del camión para reducir la velocidad, como de los responsables de la infraestructura de vías que accionan a los equipos de apoyo, como tractores y motoniveladoras para el mantenimiento de las áreas problemáticas.

Los eventos RAC son generados por la variación de presión en la suspensión de cada rueda, en donde los sensores miden los esfuerzos de presión interna, permitiendo caracterizar la condición real de las vías. El sistema recoge datos de la variación de presión en cada rueda diez veces por segundo y determina tres tipos de eventos de acuerdo con la presión de cada una: RACK (diferencia de presiones diagonales), PICTH (diferencia de presiones entre la suspensión delantera y traseras) y BIAS (diferencia de presión lateral entre la suspensión delantera y traseras).

Al integrar los SMSV e Intellimine fue posible visualizar en tiempo real los lugares donde ocurren los eventos RACK, PITCH y BIAS, y dar respuestas rápidas para corregir los puntos críticos de las vías, posibilitando operaciones más seguras tanto de los equipos como de las personas. La figura 3 muestra los puntos críticos producto del monitoreo con el sistema. 


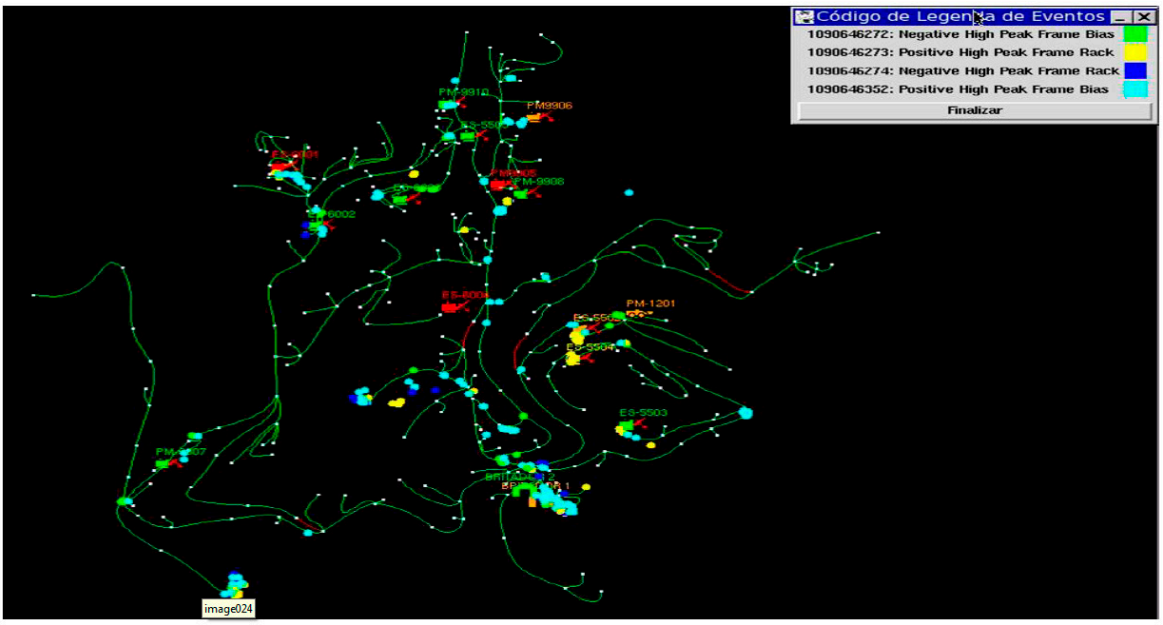

Figura 3. Mapa de la mina destacando los eventos monitoreados por el sistema RAC

Fuente: pantalla capturada en sala de monitoreo

La variación de presión y temperatura fuera de los límites puede causar daños a los neumáticos en su estructura $y$, consecuentemente, una reducción en su vida útil. Esto se debe a varios factores, como: alto número de ciclos, variación de velocidad $y$, principalmente, la calidad de las vías en las que transitan. Cuando uno de estos parámetros excede los límites podemos tener lo que llamamos desbordamiento de TKPH [(tonelada $x$ (kilómetro/hora)], donde los neumáticos ya están por encima de su punto máximo de operación y pueden producirse daños irreparables. Frente a esta situación lo ideal es verificar que estos elementos operen dentro de los límites permitidos. Con la implementación de este sistema fue posible caracterizar depresiones en las vías (figura 4) que generan aumento de TKPH y elevan las condiciones de riesgo en los neumáticos.

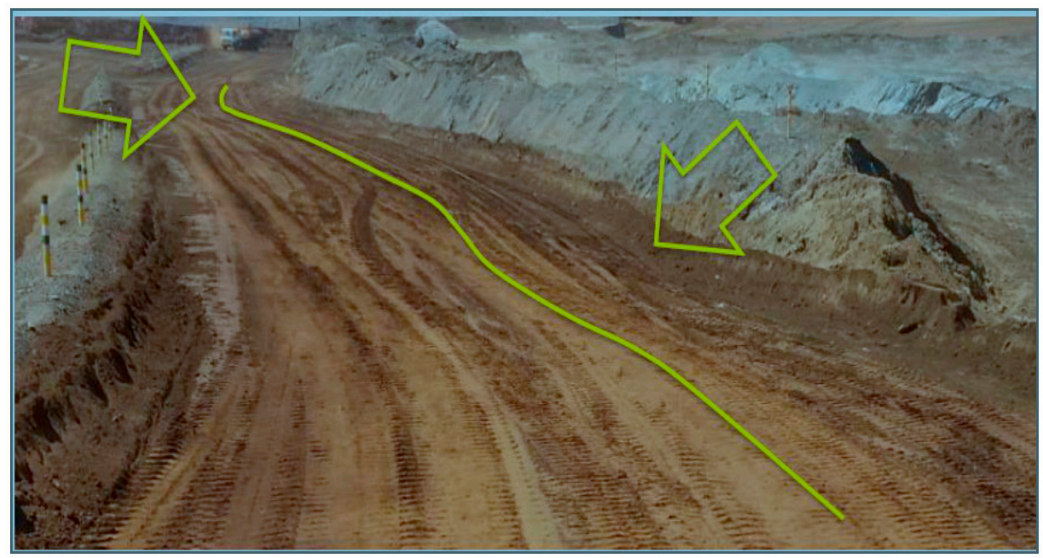

Figura 4. Depresiones en la vía

Fuente: archivo fotográfico de la mina 
Con este enfoque de gestión es posible intervenir en el proceso de manera preventiva, actuando en los puntos de riesgo que pueden ocasionar fallas funcionales. La mejora en las condiciones de las vías de acceso y plazas de carguío redunda en un mejor desempeño de indicadores de producción tales como: velocidad media, tiempo de ciclo y tiempo de maniobra.

\subsection{Monitoreo del cargamento por la herramienta RAC}

Este monitoreo permite detectar fallas en el carguío de camiones. La carga desbalanceada (figura 5) puede producir daños al chasis del equipo y sobrecarga en la suspensión y neumáticos del lado de mayor concentración de material. A medida que el equipo se mueve es sometido a fuerzas dinámicas que se agravan por las malas condiciones de las vías, generando aún más fuerza de torsión sobre la estructura del camión.

A través de las alarmas de BIAS (fuerzas transversales) de la herramienta RAC, es posible identificar cargas desbalanceadas evidenciándolas por la diferencia de presión transversal. Los analistas de flota verificaron que las alarmas de BIAS pueden ser ocasionadas por cargas desbalanceadas y no solo por condiciones impropias de las vías de acceso.

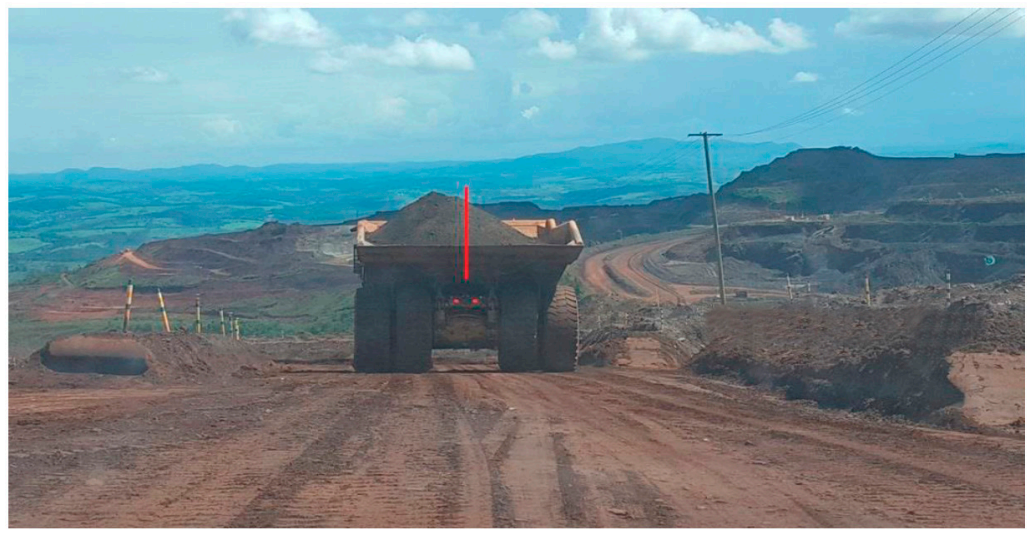

Figura 5. Carga desbalanceada

Fuente: archivo fotográfico de la mina

Este hecho contribuye considerablemente a la fatiga del chasis y los pistones hidráulicos debido a la operación excesiva, agravada aún más en el momento del basculamiento del material, donde el sistema sobrecargado sufre mayor esfuerzo para levantar la tolva del camión. Al identificar este aspecto se realizaron entrenamientos específicos con los operadores de carguío con el propósito de brindar instrucción y orientación para mantener las condiciones de operación más seguras y correctas. 


\subsection{Control de eventos de sobrecalentamiento del aceite de refrigeración de los frenos}

A través del SMSV y del sistema Intellimine, también fue posible monitorear y controlar la temperatura del fluido de refrigeración del sistema de frenos de los camiones utilizados en la mina.

Primero se realizó un análisis del perfil de las rampas y se estratificaron según su inclinación. La localización de la rampa central y la variación de inclinaciones se muestran en la figura 6.
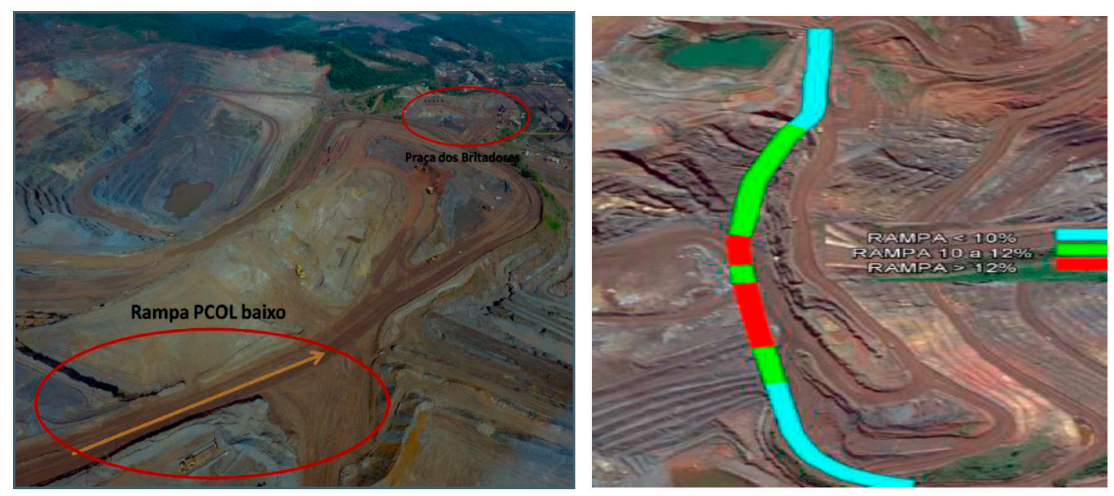

Figura 6. Rampa central de la mina con estratificación de inclinaciones

Fuente: archivo fotográfico de la mina

Además de las inclinaciones fuera de especificación por la planificación, se identificaron conductas operativas impropias como exceso de velocidad y utilización de marchas inadecuadas.

La figura 7 muestra la variación de temperatura de sistema de frenos antes y después de la realización de este trabajo. Podemos identificar un gran número de eventos de recalentamiento del fluido de refrigeración principalmente en la rampa central y en la rampa de acceso a la pila de estéril. Estos eventos generan un desgaste prematuro del sistema de frenado y pueden impactar severamente en la seguridad de las operaciones realizadas. 

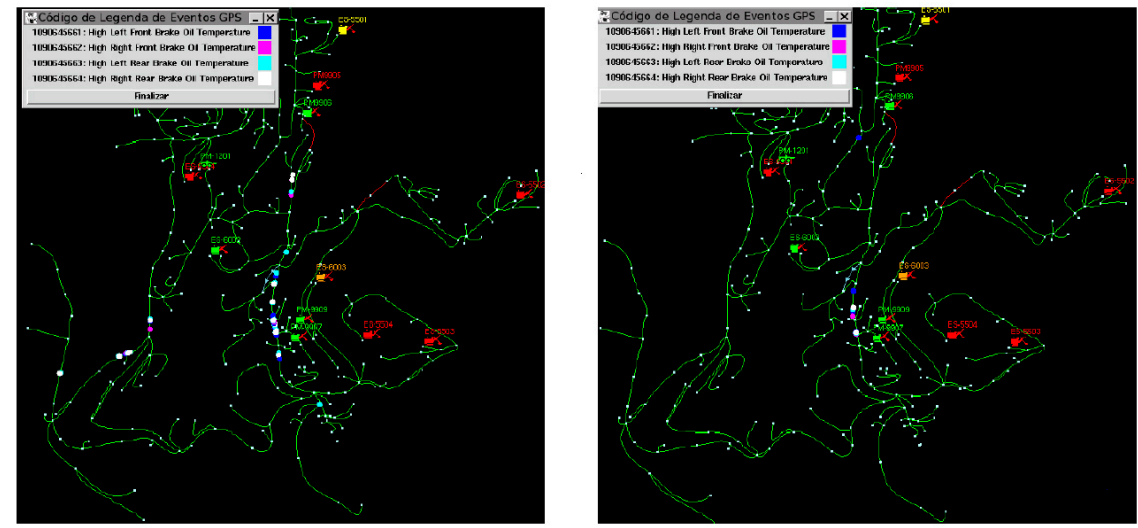

Figura 7. Eventos de recalentamiento del fluido de refrigeración de los frenos

Fuente: pantallas capturadas en sala de monitoreo

Como resultado del análisis de la información se propusieron los siguientes planes de acción para el tratamiento y reducción de los eventos de recalentamiento del fluido de refrigeración de los frenos:

- Análisis topográfico de todos los accesos a la mina.

- Mantenimiento de los accesos identificados con inclinaciones superiores al $10 \%$.

- Entrenamiento teórico y práctico de los operadores de equipos de transporte.

- Revisión de la señalización de la mina con foco en las velocidades permitidas y marchas adecuadas en cada tramo.

- Monitoreo semanal de los eventos de recalentamiento de frenos con foco en los operadores de equipos con penalizaciones administrativas en caso de reincidencias.

\section{Evaluación de resultados y beneficios de la implementación}

A partir de estos estudios fue posible constatar varios resultados relacionados con mejorías operacionales y retornos financieros. Entre ellos destacan:

a) Reducción de costos de mantenimiento debido al aumento de la vida útil de los componentes (neumáticos, chasis y suspensión). Aún no se han identificado fallas prematuras en estos ítems después de la implantación del sistema.

b) Reducción del consumo de combustible debido a la reducción de frenados y retoma de fuerza del equipo gracias a las mejores condiciones de las vías. 
c) Aumento de $15,6 \%$ en el volumen medio de diésel abastecido en la flota de transporte, comparando dos períodos específicos:

Periodo 1: entre enero y mayo del 2017 (antes de la implantación del trabajo).

Periodo 2: entre junio y diciembre del 2017.

d) La figura 8 muestra la evolución de los resultados del volumen medio y la desviación estándar de los abastecimientos.

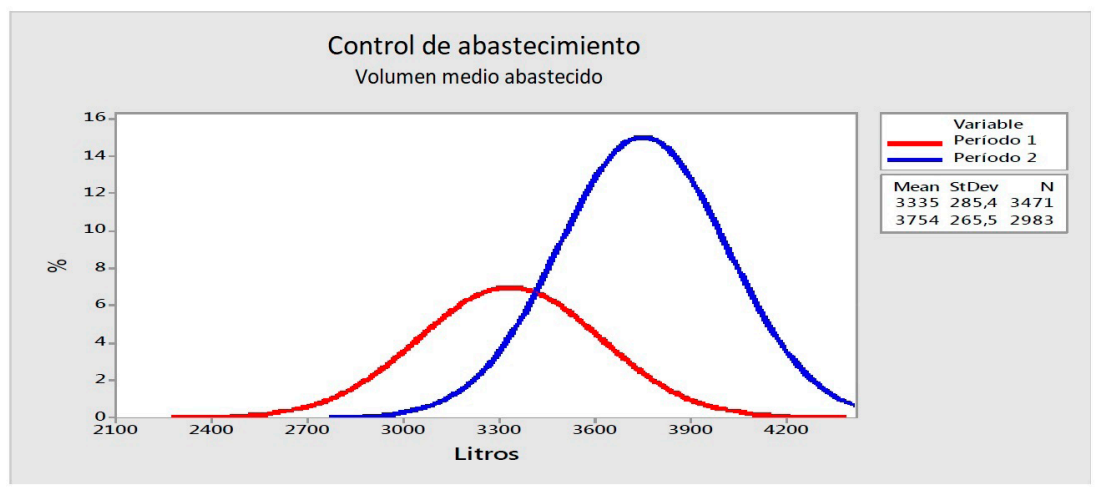

Figura 8. Análisis comparativo del volumen medio abastecido en los dos periodos analizados

Elaboración propia

e) Reducción en 21,8 \% del número medio mensual de abastecimientos por equipo de transporte (figura 9).

Promedio mensual de abastecimientos

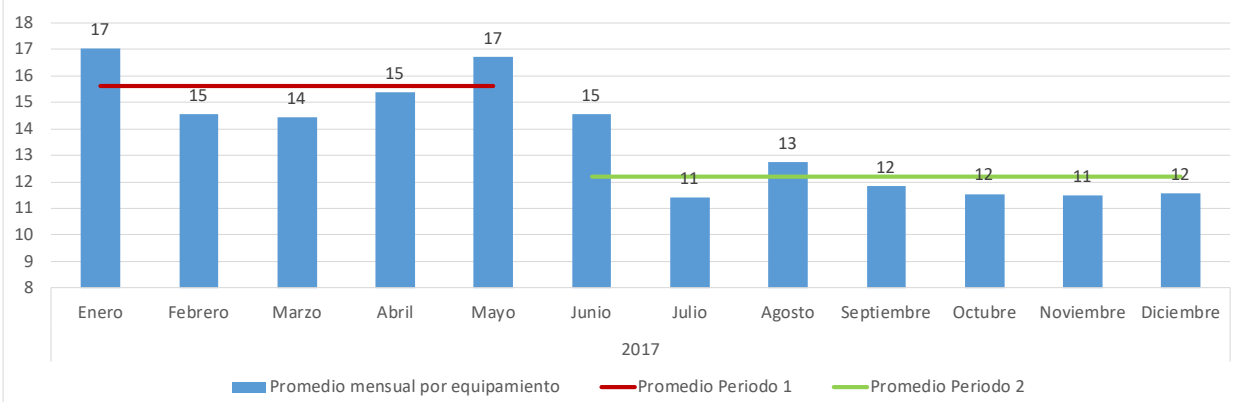

Figura 9. Promedio mensual de eventos de abastecimiento de los camiones

Elaboración propia 
f) Aumento del 0,33 \% en la utilización efectiva de la flota de transporte.

g) Priorización en el direccionamiento de equipos de infraestructura para el mantenimiento de los accesos de acuerdo con su grado crítico.

i) Reducción de eventos de TKPH agravados por irregularidades en vías, garantizando el desempeño óptimo de los neumáticos, como se muestra en la figura 10.

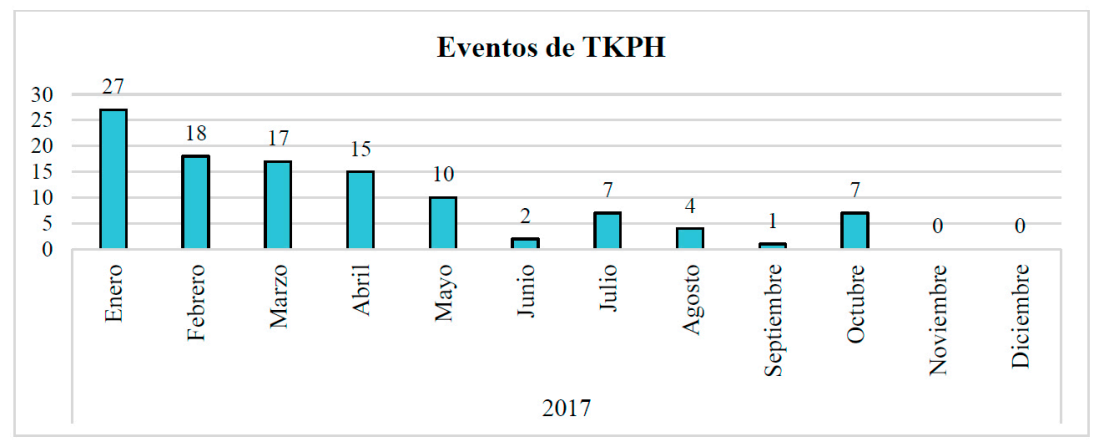

Figura 10. Evolución de los eventos de TKPH

Elaboración propia

i) Mejora de los indicadores de rendimiento operacional (productividad de transporte, velocidad media y tiempos de ciclo), como se muestra en la tabla 1.

Tabla 1. Análisis operacional del tramo CN-Machacadora

\begin{tabular}{l|c|c|c}
\hline \multicolumn{4}{c}{ Análisis operacional - trecho mina-chancado } \\
\hline Productividad efectiva (t/h) & Periodo 1 & Periodo 2 & $\Delta$ \\
\hline Tiempo promedio de ciclo (minutos) & 609,4 & 615,8 & $1,1 \%$ \\
\hline Velocidad media (km/h) & 12,6 & 12,3 & $-2,2 \%$ \\
\hline
\end{tabular}

Elaboración propia 
j) Reducción del número de eventos RACK, PITCH y BIAS (figura 11).

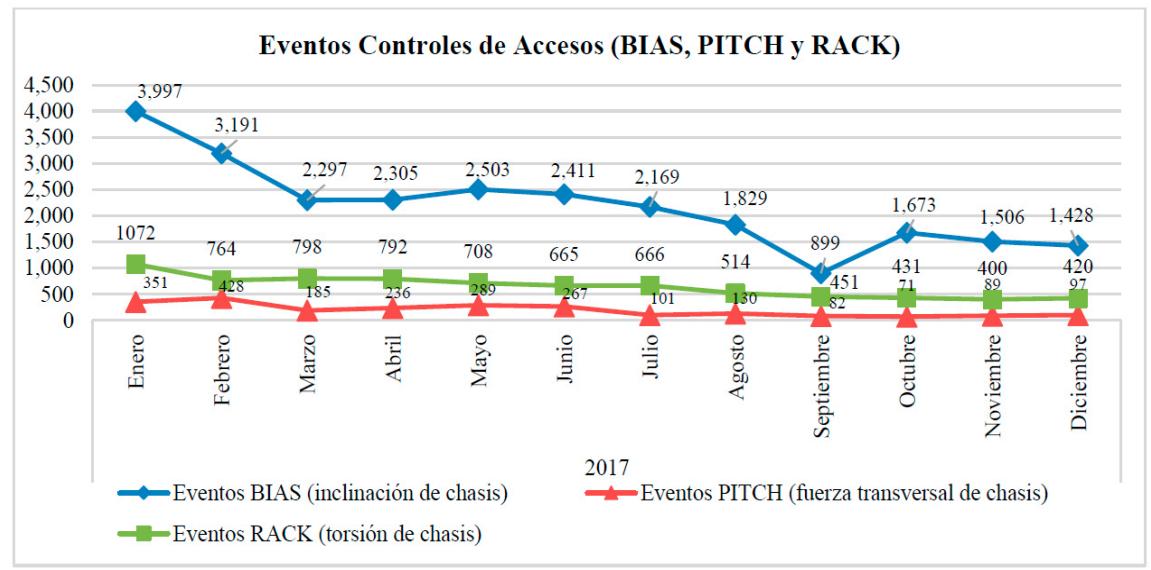

Figura 11. Evolución de los eventos RACK, PITCH y BIAS

Elaboración propia

k) Reducción del $78 \%$ en el número de eventos de recalentamiento de fluido de freno en el perfil descendente cargado (figura 12).

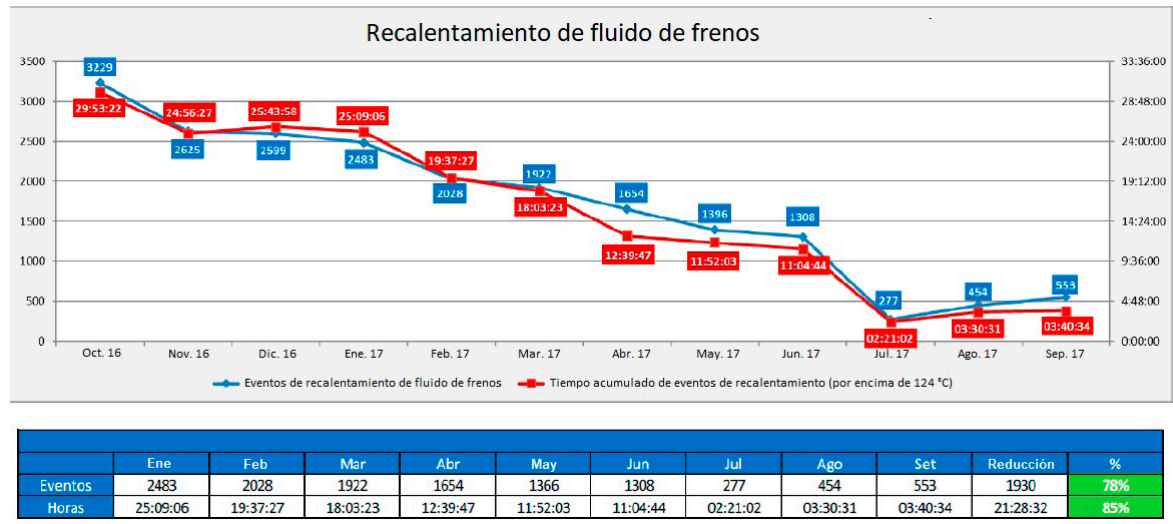

Figura 12. Comparativo de eventos de sobrecalentamiento del aceite de refrigeración de los frenos

Elaboración propia

La utilización de la telemetría en los procesos operativos posibilita un mejor gerenciamiento de las actividades en la minería. El trabajo tuvo resultados positivos en relación con equipos con mayor control en el proceso de abastecimiento y vías de acceso debidamente mantenidas, que conducen a operaciones más seguras y productivas, con menor desgaste de la 
estructura del camión, generando mayor confort y seguridad al operador, así como también un aumento en la eficiencia del proceso productivo en las operaciones de minado, y una reducción de los costos del orden del 3,3\%.

\section{Consideraciones finales}

En función del análisis de los resultados del trabajo, es posible mostrar la importancia de la implantación de la telemetría para la gestión de procesos operacionales y el control de mantenimiento en la minería. Las acciones tomadas proporcionan un aumento en la confiabilidad y disponibilidad de los equipos, contribuyendo a la sostenibilidad del negocio. El monitoreo de los procesos es un factor determinante para el cambio cultural vivido por los involucrados en la cadena de producción, lo que significa que la unión del uso de la tecnología y factor humano son los ingredientes clave para la conquista de los resultados.

Se justifica así la necesidad de utilizar nuevas tecnologías de información, que tienen por objeto identificar, entender, evitar o minimizar los puntos débiles y posibles problemas que están causando resultados no deseados en una organización. Con los datos obtenidos a través de la telemetría es posible que haya una gestión eficiente, donde los recursos serán optimizados y utilizados con mayor eficacia, mejorando significativamente el rendimiento de los equipos y del proceso productivo.

\section{Referencias}

Cochefski, P. J. (2011). Fuel management: automated services can be a boon to the bottom line for truck fleets fueling facilities. Bulk Transporter, 74(5).

Kardec, A., y Dorigo, L. (2009). Manutenção orientada para resultados. Rio de Janeiro: Qualitymark.

Kardec, A., y Nascif, J. (2009). Manutenção: função estratégica (3.a edición). Rio de Janeiro: Qualitymark.

Knights, P. F., y Daneshmend, L. K. (2000). Open systems standards for computing in the mining industry. CIM bulletin, 89-92.

Lewis, M. W., y Steinberg, L. (2001). Maintenance of mobile mine equipment in the information age. Journal of Quality in Maintenance Engineering, 7(4), 264-274.

Tannant, D., y Regensburg, B. (2001). Guidelines for mine haul road design. Alberta: School of Mining and Petroleum Engineering. Department of Civil and Environmental Engineering. University of Alberta. 
Thompson, R., y Visser, A. (1997). A mechanistic structural design procedure for surface mine haul roads. International Journal of Surface Mining, Reclamation and Environment, 11(3), $121-128$.

Thompson, R., y Visser, A. (2000). The functional design of surface mine haul roads. Journal of the South African Institute of Mining and Metallurgy100(3), 169-180.

Thompson, R., y Visser, A. (2003). Mine haul road maintenance management systems. Journal of the South African Institute of Mining and Metallurgy 103(5), 303-312.

Thompson, R. J., y Visser, A. (2006). The impact of rolling resistance on fuel, speed and costs. Continuous improvement case study, 2(1), 68-75. 